\title{
Simultaneous Multislice Refocusing via Time Optimal Control
}

\author{
Armin Rund $d^{*}+\frac{\hbar}{+}$ \\ Christoph Stefan Aigner ${ }^{\S} \div$ \\ Karl Kunisch ${ }^{*}$ I \\ Rudolf Stollberger ${ }^{\S, \dagger}$
}

Purpose: Joint design of minimum duration RF pulses and slice-selective gradient shapes for MRI via time optimal control with strict physical constraints, and its application to simultaneous multislice (SMS) imaging.

Theory and Methods: The minimization of the pulse duration is cast as a time optimal control problem with inequality constraints describing the refocusing quality and physical constraints. It is solved with a bilevel method, where the pulse length is minimized in the upper level, and the constraints are satisfied in the lower level. To address the inherent nonconvexity of the optimization problem, the upper level is enhanced with new heuristics for finding a near global optimizer based on a second optimization problem.

Results: A large set of optimized examples shows an average temporal reduction of $87.1 \%$ for double diffusion and $74 \%$ for turbo spin echo pulses compared to PINS pulses. The optimized results are validated on a 3T scanner with phantom measurements.

Conclusion: The presented design method computes minimum duration RF pulse and slice-selective gradient shapes subject to physical constraints. The shorter pulse duration can be used to decrease the effective TE in existing EPI or echo spacing in TSE sequences.

Keywords: pulse design, refocusing, time optimal control, simultaneous multi-slice excitation

\section{Introduction}

Simultaneous Multislice (SMS) imaging acquires the information of different slices simultaneously to reduce the overall acquisition time and is an emerging imaging technique with various applications such as multi-directional diffusion tensor imaging [39], short echo time (TE) functional imaging [11] and clinically important turbo spin echo (TSE) based sequences [16]. Contrary to pure in-plane accelerated parallel imaging, SMS acceleration in slice direction acquires signal for every extra slice measured and has only a minimal signal-to-noise penalty [6]. Recently, the application of Wave-CAIPI [8], originally proposed for $3 \mathrm{D}$ encoding, to SMS imaging allowed an increase in the number of simultaneous slices, called the multiband (MB) factor, to 13 without major g-factor penalties [16]. On the other hand, SMS refocusing pulses with a low $\mathrm{MB}$ factor but a good refocusing profile are beneficial for high resolution diffusion imaging $[20,38,41]$.

\footnotetext{
*Institute for Mathematics and Scientific Computing, University of Graz, Heinrichstrasse 36, 8o1o Graz, Austria (armin. rund@uni-graz . at)

†BioTechMed Graz, 8o10 Graz, Austria

† The first two authors equally contributed to the paper

§Institute of Medical Engineering, Graz University of Technology, Stremayrgasse 16/III, 8010 Graz, Austria

IJohann Radon Institute for Computational and Applied Mathematics (RICAM), Austrian Academy of Sciences, Altenbergstraße 69, 4040 Linz, Austria
} 
However, SMS imaging requires RF pulses that simultaneously excite or refocus slices, which typically result in RF amplitudes and power which are too high, or in very long pulse durations [6]. Historically, the first SMS RF pulse design method is based on a superposition of frequency shifted sub-pulses [32]. To overcome the limitation of a linear scaling of the $B_{1}$ peak amplitude and overall RF power with the MB factor $[6,30]$, different groups proposed design methods via phase modulation [45], time shifted superposition [5], root-flipping [41] and use of the wavelet domain [24]. After the pulse design, additional $B_{1}$ and power reduction can be achieved by applying variable-rate selective excitation (VERSE) $[14,21,4 \mathrm{O}]$. However, high MB factors lead to a strong oscillation of the RF amplitude and a direct VERSE application might require additional smoothing of the otherwise too rapidly changing gradient waveform, which was resolved by applying VERSE on the overall RF pulse envelope [28] Alternatively, an inherent power reduction in the pulse design is possible using the power independent number of slices (PINS) [33] method that utilizes periodic excitation patterns, with the limitation that a low time-bandwidth product (TBP) is generally used in order to avoid long pulse durations.

The combination of PINS pulses with conventional SMS pulses, MultiPINS [15], combines both approaches to reduce the overall pulse duration and increase the bandwidth of PINS pulses.

In addition, SMS RF pulses can also be designed by optimal control methods. Optimal Control (OC) has proven to be successful in NMR and MRI to design accurate excitation profiles based on different models such as the Liouville-von Neumann equation [12, 27, 42], the Bloch equations $[3,4,13,44]$, or the Bloch spin-domain model [19].

To encourage researchers to focus on new methods that compute short SMS pulses with a low $B_{1}$ peak and SAR, while achieving accurate excitation/refocusing profiles, SMS pulse design was chosen to be the topic of the 2015 ISMRM challenge [18] (October 2015 to May 2016, http: //challenge .ismrm. org/node/71). The method described in this paper was developed and applied in its SMS design subchallenge.

In our preceding work [36,37] we introduced an optimal control method for RF pulse design subject to different equality and inequality constraints using a fixed time horizon. In contrast, we present here a new time optimal control modeling for designing RF pulses with minimum duration under technical constraints. Due to the free terminal time, the solution of time optimal control problems [22] is algorithmically involved and different methods are needed. We introduce a new bilevel method with time grid adaption and warm-start algorithms. Moreover, globalization plays a central role, since both the free terminal time and the bilinear Bloch equation lead to a nonconvex optimization problem. Here, we introduce new heuristics for finding a near global optimizer. The optimization method is tested on 31 SMS refocusing examples. The numerical results are validated by experimental phantom measurements on a 3 T MR system.

\section{Theory}

In the following, refocusing in minimum time is posed as a time optimal control problem with inequality constraints for slice profile fidelity and MR hardware restrictions. The constraints were inspired by the ISMRM Challenge [18].

\subsection{Optimal control framework}

We jointly optimize for the time horizon/pulse duration $T$, as well as the RF pulse and slice-selective gradient (Gs) slew rate values at each time sample. Applying an equidistant time grid $t_{m}=m \tau, m=$ $0, \ldots, N_{t}$ with step size $\tau=T / N_{t}$, the RF pulse is described as $B_{1, m}=r_{m} \exp \left(i \vartheta_{m}\right)$ with amplitude $r_{m}$, phase $\vartheta_{m}$ and the imaginary unit $i$. The Gs amplitude $G_{m}$ is given via the slew rate $s_{m}$ as

$$
G_{m}=G_{m-1}+\tau s_{m-1}, \quad m=2, \ldots, N_{t}-1
$$


with given boundary conditions $G_{1}, G_{N_{t}}$. The Bloch equation is solved exactly in the spin-domain, see $[26,34]$, neglecting relaxation effects, which is reasonable for short $T$. The evolution of the magnetization is described by the complex-valued Cayley-Klein parameters

$$
a_{m}=\alpha_{m} a_{m-1}-\beta_{m}^{*} b_{m-1}, \quad b_{m}=\beta_{m} a_{m-1}+\alpha_{m}^{*} b_{m-1},
$$

for $m=1, \ldots, N_{t}$ with $a_{0}=1, b_{0}=0$, the gyromagnetic ratio $\gamma$, and coefficients

$$
\begin{aligned}
& \alpha_{m}=\cos \left(\phi_{m} / 2\right)+i \gamma \tau z G_{m} \sin \left(\phi_{m} / 2\right) / \phi_{m}, \\
& \beta_{m}=i \gamma \tau B_{1, m} \sin \left(\phi_{m} / 2\right) / \phi_{m}, \\
& \phi_{m}=-\gamma \tau \sqrt{r_{m}^{2}+\left(z G_{m}\right)^{2}} .
\end{aligned}
$$

Therein, the spatial point $z$ covers the field of view (FOV) $\Omega$ which is discretized equidistantly as $z_{j}, j=1, \ldots, N_{z}$ with the spatial resolution $\delta . \Omega$ is separated into the in-slice domain $\Omega_{\text {in }}=\cup_{l=1}^{N_{\mathrm{MB}}} S_{l}$ containing the interior $S_{l}$ of each of the $N_{\mathrm{MB}}$ slices , the out-of-slice domain $\Omega_{\text {out }}$, and a free transition region in between. For refocusing in minimum time we optimize the control $\mathbf{x}=\left(r_{1}, \ldots, r_{N_{t}}\right.$, $\vartheta_{1}, \ldots, \vartheta_{N_{t}}, s_{1}, \ldots, s_{N_{t}-2}$ ) and the pulse duration $T$ in order to

$$
\min _{T>0, \mathbf{x}} T \quad \text { subject to (1), }
$$

(2) for $z=z_{j}, j=1, \ldots, N_{z}$,

$$
\begin{aligned}
& \left|b_{N_{t}}\right|^{2}-1 \leq e \quad \text { for } z_{j} \in \Omega_{\text {in }}, \quad\left|b_{N_{t}}\right|^{2} \leq e \quad \text { for } z_{j} \in \Omega_{\text {out }}, \\
& \left|\varphi-\bar{\varphi}_{l}\right| \leq e_{p}, \quad \text { for } z_{j} \in S_{l}, \quad l=1, \ldots, N_{\mathrm{MB}},
\end{aligned}
$$$$
0 \leq r_{m} \leq r_{\max }, \quad\left|s_{m}\right| \leq s_{\max }, \quad-\pi \leq \vartheta_{m} \leq \pi,
$$

$$
\begin{aligned}
& \left|G_{m}\right| \leq G_{\max }, \quad\left|\frac{G_{N_{t}}-G_{N_{t}-1}}{\tau}\right| \leq s_{\max }, \\
& \mathrm{SAR}_{\mathrm{e}}=\operatorname{SAR}_{\text {coileff }} f_{p} \tau \sum_{m=1}^{N_{t}} r_{m}^{2} \leq S A R_{\max } .
\end{aligned}
$$

The inequalities model the slice profile accuracy in magnitude and phase, amplitude and SAR constraints. In (5) the slice profile is prescribed with a tolerance $e>0$ (that may depend on the spatial location) around a perfect refocusing. Here, ideal crusher gradients are assumed to completely dephase the free induction decay produced by the refocusing pulse $[7,34]$. In (6) the phase $\varphi=\arg \left(b_{N_{t}}^{2}\right)$ is allowed to vary only up to a tolerance $e_{p}=e_{p}\left(z_{j}\right)>0$ from the arithmetic mean $\bar{\varphi}_{l}$ of the phase in slice $S_{l}$. MR hardware restrictions are included as amplitude constraints in (7), (8) with maximum amplitudes $r_{\max }, G_{\max }, s_{\max }>0$. The last inequality in (8) incorporates the given terminal condition $G_{N_{t}}$. Since a low SAR and a short pulse duration are competing goals, a SAR limit $S A R_{\max }>0$ is prescribed. For single transmit MR imaging, it is here reasonable to apply a SAR estimate $\mathrm{SAR}_{\mathrm{e}}\left(\mathrm{W} \mathrm{kg}^{-1}\right)$ based on the global pulse power with constant $\mathrm{SAR}_{\text {coileff }}\left(\mathrm{W} / \mathrm{kg} / \mu \mathrm{T}^{2}\right)$ and constant pulse rate $f_{p}$.

This optimization problem is a pure time optimal control problem with control and state constraints. Such problems tend to possess bang-bang solutions, where the control constraint is active all the time but the value jumps between the upper and lower bound, see e.g. [22]. To approximate such optimal controls reliably we suggest an $L^{2}$-regularization of the controls with parameters $\alpha, \zeta>0$. Furthermore, pointwise state-constrained optimal control problems are known to be involved since the Lagrange multiplier is irregular which leads to a decrease of the convergence speed and accuracy of numerical solution methods, see e.g. [23]. To reduce these effects we apply the $L^{p}$-penalization of the state constraints from [36] and drive the exponent $p \rightarrow \infty$ in a homotopy loop as we approach the 
optimizer. Therefore, we define the penalized objective

$$
\begin{aligned}
\min _{T>0, \mathbf{x}} J & =T+\frac{\tau}{2} \sum_{m=1}^{N_{t}} \alpha r_{m}^{2}+\zeta \vartheta_{m}^{2}+\frac{\zeta \tau}{2} \sum_{m=1}^{N_{t}-2} s_{m}^{2}+\frac{\delta \mu_{\mathrm{out}}}{2 p} \sum_{z_{j} \in \Omega_{\mathrm{out}}}\left(\frac{\left|b_{N_{t}}\right|^{2}}{e}\right)^{p}+\frac{\delta \mu_{\mathrm{in}}}{2 p} \sum_{z_{j} \in \Omega_{\mathrm{in}}}\left(\frac{\left|b_{N_{t}}\right|^{2}-1}{e}\right)^{p} \\
& +\frac{\delta \mu_{\mathrm{p}}}{p} \sum_{l=1}^{L} \sum_{z_{j} \in S_{l}}\left(\frac{\varphi-\bar{\varphi}_{l}}{e_{p}}\right)^{p}+\frac{\tau \mu_{w}}{p} \sum_{m=2}^{N_{t}-1}\left(\frac{G_{m}}{G_{\max }}\right)^{p}+\frac{\mu_{T}}{p}\left(\frac{G_{N_{t}}-G_{N_{t}-1}}{\tau s_{\max }}\right)^{p}
\end{aligned}
$$

with even number $p$ and parameters $\mu_{\text {out }}, \mu_{i n}, \mu_{p}, \mu_{w}, \mu_{T}>0 . \alpha$ is automatically adapted to fulfill the SAR constraint (9), $\zeta$ is decreased as the optimizer is approached. This penalized objective has to be minimized subject to (1), (2), (9), and the pointwise control constraints (7). For a fixed $T$ this problem can be solved with established methods of numerical optimal control. We apply the trust-region semismooth quasi-Newton method from [36], which features robustness and adjoint-based exact discrete derivatives. It also handles the automatic adaption of the penalty parameters $\mu_{\text {out }}, \mu_{i n}, \mu_{p}, \mu_{w}, \mu_{T}$. However, we still have to take care of the free terminal time $T$.

\subsection{Bilevel method for time optimal control}

Time-optimal control problems can be solved by different approaches. In our approach, the control $\mathbf{x}$ and $T$ are separated in a bilevel method, where $T$ is kept fixed in the lower level problem. Alternatively, both can be treated at once using time transformations, see e.g. [29]. For the Bloch equation on equidistant time grids, numerical studies showed that a bilevel method prevails, since it facilitates keeping a good slice profile pattern after a time reduction. Furthermore, it offers more flexibility in the time reduction, which is exploited for finding an improved minimizer.

The bilevel method is initialized using an existing method for SMS pulse design, e.g. conventional superposition [32], phase scrambling [45], root-flipped pulses [41], PINS [33] or MultiPINS [15]. Then, we alternately reduce the terminal time (upper level) and fulfill the constraints (lower level). For the former we keep the time step constant and reduce the terminal time by deleting one time point. The latter is done by minimization of the penalized objective (10) for a fixed $T$ using the trust-region semismooth quasi-Newton method of [36] with the following changes. First, we do not fully iterate until a relative or absolute stopping criteria is fulfilled, but terminate as soon as we have found an admissible solution to (5)-(9). Second, $p$ is not changed during the course of the semismooth quasiNewton method, but altered in the upper level of the bilevel method in order to have a monotonicity of $p$ towards the optimum. Sometimes in the alternation of lower and upper level we decide to increase $p$ and might apply a refinement of the time grid (depending on the allowed raster time). The resulting bilevel method reads:

Step 1: Initialize control $\mathbf{x}_{0}$ and duration $T_{0}$ with a conventional RF pulse design method, choose $p$ and set $n=1$.

Step 2: Upper level: Choose to delete one time instance $t_{k}$ applying the heuristics for an improved optimizer (11). Reduce the terminal time to $T_{n}<T_{n-1}$ and warm-start $\mathbf{x}_{n}=f\left(\mathbf{x}_{n-1}, k\right)$.

Step 3: Lower level: Minimize the penalized objective (10) for a fixed terminal time $T_{n}$ by a trustregion semismooth quasi-Newton method until an admissible solution to the inequality constraints (5)-(9) is found. The resulting control is $\mathbf{x}_{n}$.

Step 4: Decide to increase $p$, decrease $\zeta$ and/or to refine the time grid. Set $n=n+1$ and repeat from Step 2.

Finally, we present the technical details of the time reduction. At deletion of a time point $t_{k}$, the current control $\mathbf{x}_{n-1}$ is represented on the new time grid $\mathbf{x}_{n}=f\left(\mathbf{x}_{n-1}, k\right)$ using a transfer function $f$ that performs a good warm-start for the next lower level optimization. In particular, for maintaining a good slice profile this transfer function distributes the values of $G_{k}$ and the real and imaginary part 
of $B_{1, k}$ at the deleted time instance $t_{k}$ symmetrically to the neighboring time instances. While doing so, we fulfill the constraints (7), (8). For technical details of $f$ we refer to its source code, which we published together with the software (see below). The time point $t_{k}$ to be deleted is chosen based on new heuristics for finding a near global optimizer. We choose the time point that allows the best performance after deletion, warm-start and $N_{\mathrm{g}}$ steps of the lower level solver. More precisely, the time $t_{k}$ is determined as the global solution to the optimization problem

$$
\min _{m \in\left\{1, \ldots, N_{t}{ }^{n}\right\}} J\left(T_{n}, Q\left(f\left(\mathbf{x}_{n-1}, m\right)\right)\right),
$$

where $Q$ is an abstract function that stands for solving the lower level problem with the trust-region semismooth quasi-Newton method of [36] in at most $N_{\mathrm{g}}$ iterations. Obviously, a larger $N_{\mathrm{g}}$ gives a better minimizer at the end, but increases the runtime of the code. We use $N_{\mathrm{g}}=0$ for fast runs and $10 \leq N_{\mathrm{g}} \leq 20$ otherwise. Then the global minimizer of this auxiliary problem is computed exactly by total enumeration, or approximately by reusing information from previous upper level steps.

\section{Methods}

\subsection{Simulations and pulse design}

To test the general applicability of the proposed design method, we minimized the pulse duration in the test set of 31 cases given by the organizers of the ISMRM Challenge [18]. It contains different problem parameters (i.e. MB factor and slice thickness (THK)) and 13 different constraints (i.e. $B_{1}$ peak, slew rate of Gs, maximum refocusing errors and SAR limits). For the sake of completeness the most important problem parameters and constraints are repeated below. A full description is given by the ISMRM 2015 challenge homepage and [18].

The maximum refocusing error $e$ was set to 0.02 out-of-slice and 0.03 in-slice. For the design of all SMS refocusing pulses we assumed perfect crusher gradients. The space was discretized equidistantly with a resolution $\delta=T H K / 400$. Two different example classes were considered for the optimization, double refocused diffusion and TSE/RARE imaging, each with different problem parameters (see Supporting Table $\mathrm{S}_{1}$ ). The global SAR constraint for all cases was set to be $S A R_{\max }=3.2 \mathrm{~W} \mathrm{~kg}^{-1}$. The SAR estimate $S_{A R}$ used an expected SAR efficiency of a $3 T$ birdcage coil SAR coileff of $0.25 \mathrm{~W} / \mathrm{kg} / \mu \mathrm{T}^{2}$ and the assumed pulse rate $f_{p}$ for the two different scenarios (see Supporting Table $\mathrm{S} 1$ ). The influence of amplitude constraints on the optimization was analyzed by using three different constraint settings given by actual hardware limits (see Supporting Table S2).

Diffusion pulses (DIFF). This example class asked for refocusing pulses for double refocused diffusion sequences [35] with a small MB factor (3-5) and THK (1-2 mm), see Supporting Table S1. The number of spatial points $N_{z}$ varied from $2401(\mathrm{MB}=3$, THK $=2 \mathrm{~mm})$ to $4801(\mathrm{MB}=5$, THK $=1 \mathrm{~mm})$ to define a FOV of $120 \mathrm{~mm}$. The SAR estimate $\mathrm{SAR}_{\mathrm{e}}$ used a repetition time (TR) of $120 \mathrm{~ms}$ with two identical refocusing pulses per repetition, resulting in a pulse rate $f_{p}=16.67 / \mathrm{s}$. The phase of the refocusing profile was not considered in the optimization.

Turbo spin echo pulses (TSE). Here the task was to generate SMS refocusing pulses with a large number of simultaneous and thin slices $(\mathrm{MB}=8-14$, THK $=0.5-2 \mathrm{~mm}$, TBP $=3)$ for a repeated application in a TSE/RARE based sequence. A phase constraint with a maximal deviation of $e_{p}=0.01$ radiant from the mean phase per slice was added according to (6). The bigger FOV of $240 \mathrm{~mm}$ resulted in more degrees of freedom in the spatial direction, ranging from $4801(\mathrm{MB}=8$, THK $=2 \mathrm{~mm})$ up to $19201(\mathrm{MB}=14$, THK $=0.5 \mathrm{~mm})$. For all TSE cases a turbo factor of 12 and a TR of $220 \mathrm{~ms}$ was assumed resulting in a pulse rate $f_{p}=54.55 / \mathrm{s}$.

The optimization was in general started from RF and Gs waveforms based on the PINS method [33]. The sub-pulse envelope was computed by the SLR algorithm [34] using $d_{1}=e / 4$ (in-slice) and $d_{2}=e / \sqrt{2}$ 
(out-of-slice). For $\tau=10 \mu$ s the initial pulse durations $T_{\text {init }}$ ranged from $8.710 \mathrm{~ms}$ ( $\mathrm{MB}=14$, THK $=2$ $\mathrm{mm})$ to $32.42 \mathrm{~ms}(\mathrm{MB}=8, \mathrm{THK}=0.5 \mathrm{~mm})$, see Table 1 and 2 .

The optimization method was implemented in MATLAB (The MathWorks, Inc, Natick, USA). A version of it can be downloaded from https://github.com/rundar/mr. control. Initially a small penalty exponent $p=6$ was chosen, which was doubled towards the optimum gradually to about $10^{3}$ to $10^{4}$ based on previous experience [36]. The trust-region semismooth quasi-Newton method [36] calibrated and adapted the other parameters in the objective (10) automatically. The time step size was initially set to $\tau=10 \mu \mathrm{s}$, and gradually decreased by a factor of two as long as the optimizer stayed below 20000 sampling points. For the challenge we typically chose $N_{\mathrm{g}}=20$ for the auxiliary optimization problem of the best time reduction, and reused the information several times. All calculations were done in parallel on the high-performance-computing-cluster "RADON 1" (RICAM, Linz, Austria) using one node ( $2 \times$ Xeon E5-2630v3 with in total 16 cores and 128 GB of RAM) for each case. Two examples were analyzed and described in more detail. One diffusion case with $\mathrm{MB}=3$ and $\mathrm{THK}=1.75 \mathrm{~mm}$ and one TSE/RARE case with $\mathrm{MB}=12$ and THK $=1 \mathrm{~mm}$.

\subsection{Experiments}

To validate the numerical simulations, phantom measurements were performed on a 3T MR scanner (Magnetom Skyra, Siemens Healthcare, Erlangen, Germany) using the transmit/receive body-coil (birdcage). A standard crushed spin echo (SE) sequence was modified to import arbitrary RF and Gs shapes, and to measure the slice profile by changing the phase encoding to the slice direction. We created three SE sequences, non-selective excitation and refocusing, slice-selective MB excitation with non-selective refocusing, and slice-selective $\mathrm{MB}$ excitation with optimized slice-selective $\mathrm{MB}$ refocusing (applying the proposed optimization method). We implemented two optimized refocusing pulses and slice-selective gradient shapes, both shown in Figure 3 ("scanner 2"), optimized for the hardware constraints that comply with the MR system used. Their durations were $T=3.38 \mathrm{~ms}$ (DIFF MB3) and $T=5.78 \mathrm{~ms}$ (TSE MB12) using a temporal grid of $10 \mu$ s equivalent to the gradient raster time of the MR system.

The utilized non-selective rectangular block pulses were $0.8 \mathrm{~ms}$ long for both, excitation and refocusing. The slice-selective MB excitation pulses were created applying superposition with phase shifted SLR sub-pulse envelopes [34] resulting in a pulse duration of $T=5.58 \mathrm{~ms}$ and a constant slice-selective gradient of $10 \mathrm{mT} / \mathrm{m}$ for the DIFF MB3 case. For the TSE MB12 case we have $T=10.92 \mathrm{~ms}$ and 5 $\mathrm{mT} / \mathrm{m}$.

The experiments were performed using a spherical phantom with a diameter of $240 \mathrm{~mm}$ filled with $0.011 \mathrm{~g}$ MACROLEX blue per liter MARCOL-oil ( $T_{1} \approx 200 \mathrm{~ms}$ and $\left.T_{2} \approx 100 \mathrm{~ms}\right)$. High resolution data were acquired in the transversal plane with a matrix size of $1536 \times 1536$ (1536 phase encoding steps) and a FOV of $300 \times 300 \mathrm{~mm}$ resulting in a voxel size of $0.2 \times 0.2 \mathrm{~mm}$. For both cases we used TR $=300 \mathrm{~ms}$.

Table 1: Comparison of the optimized pulse duration (in ms) with the duration of the PINS initial guess for all DIFF examples.

\begin{tabular}{ccccccc}
\multicolumn{4}{c}{$\mathrm{MB}_{3}$} & \multicolumn{2}{c}{$\mathrm{MB}_{4}$} & \multicolumn{2}{c}{$\mathrm{MB}_{5}$} \\
\hline $\begin{array}{c}\text { THK } \\
\mathrm{mm}\end{array}$ & $\begin{array}{c}\text { PINS } \\
\mathrm{ms}\end{array}$ & $\begin{array}{c}\text { opt } \\
\mathrm{ms}\end{array}$ & $\begin{array}{c}\text { PINS } \\
\mathrm{ms}\end{array}$ & $\begin{array}{c}\text { opt } \\
\mathrm{ms}\end{array}$ & $\begin{array}{c}\text { PINS } \\
\mathrm{ms}\end{array}$ & $\begin{array}{c}\text { opt } \\
\mathrm{ms}\end{array}$ \\
\hline 2.00 & $16.87 \mathrm{O}$ & 2.155 & 15.090 & 2.285 & 13.560 & 2.414 \\
1.75 & $18.46 \mathrm{O}$ & 2.155 & 16.400 & 2.448 & $14.14 \mathrm{O}$ & 2.502 \\
$1.5 \mathrm{O}$ & $21.26 \mathrm{O}$ & 2.336 & 18.470 & 2.539 & $16.09 \mathrm{O}$ & 2.651 \\
1.25 & $24.37 \mathrm{O}$ & $2.47 \mathrm{O}$ & $21.18 \mathrm{O}$ & 2.673 & $18.32 \mathrm{O}$ & $2.4 \mathrm{O} 4$ \\
1.00 & $28.84 \mathrm{O}$ & $2.65 \mathrm{O}$ & $25.25 \mathrm{O}$ & 2.805 & $21.18 \mathrm{O}$ & 2.938 \\
\hline
\end{tabular}


The TE was set to $23 \mathrm{~ms}$ (DIFF MB3) and $30 \mathrm{~ms}$ (TSE MB12) for both the non-selective and optimized SE experiments. The TE of the fully non-selective SE was set to $15.5 \mathrm{~ms}$. All measurements were acquired after a manual shim with a sampling bandwidth of $130 \mathrm{~Hz}$ and were repeated five times to compute the median with an increased signal to noise ratio. The experimental data using the optimized slice-selective refocusing were normalized by the fully non-selective SE measurement using a masked noise cut-off of 0.1 .

\section{Results}

Below we present the results of our contribution to the ISMRM challenge. In particular, we show the real-valued $\mathrm{RF}$ pulses ( $B_{1, m} \in \mathbb{R}$ for all times) which were submitted for the challenge.

\subsection{Simulations}

Supporting Figure $S 1$ summarizes the numerical results of an optimized diffusion example to refocus 5 slices with a thickness of $1.25 \mathrm{~mm}$ equally distributed over a FOV of $120 \mathrm{~mm}$ at a temporal resolution of $\tau=0.625 \mu \mathrm{s}$. The overall terminal time could be reduced to $2.404 \mathrm{~ms}$, which is a reduction of $86.9 \%$ compared to the initial PINS pulse with $18.32 \mathrm{~ms}$ and a reduction of $90.3 \%$ compared to a valid pulse candidate based on conventional superposition with $24.85 \mathrm{~ms}$ (not shown). The optimized RF amplitude and slew rate are given in the first row of Supporting Figure S1. The second row additionally shows the Gs shape, and the simulated refocusing profile for the whole FOV. A zoomed image in the third row shows, that the profile always remains inside the black error corridor. The last plot shows the phase of each refocusing profile, which was not constrained here.

Figure 1 shows the analogous plots, this time for the optimized TSE refocusing (MB12, THK $=1 \mathrm{~mm}$, $\mathrm{FOC}=240 \mathrm{~mm}, \tau=0.625 \mu \mathrm{s})$. The optimization was done here without a constraint on the SAR. The pulse duration was reduced by $75 \%$ from $12.92 \mathrm{~ms}$ to $3.16 \mathrm{~ms}$ compared to the initial PINS pulse. Again, the gradient amplitude in the third plot shows a large hump at the beginning and the end, and a small zigzagging in between that remained from the initial PINS pulse. The slice profile fulfills the constraints, with an equiripple error in the out-of-slice region, which can be seen in the zoomed plot in the third row for one slice. The last plot shows the additional phase constraint for the TSE/RARE examples. The refocusing phase is nearly constant per slice, but each slice shows a different mean phase.

A summary for the total pulse duration of the PINS based initial guesses $T_{\text {init }}$ compared to the time optimal pulse durations $T$ is given in Table 1 (DIFF) and Table 2 (TSE). Here, all 31 examples were solved with the SAR constraint. The achieved temporal reduction for the diffusion example is $86.9 \%$ (from $18.32 \mathrm{~ms}$ to $2.404 \mathrm{~ms}$ ) and for the TSE example $71 \%$ (from $14.21 \mathrm{~ms}$ to $4.12 \mathrm{~ms}$ ). Overall, an average reduction of $87.1 \%$ could be achieved for all diffusion cases and $74 \%$ for all TSE cases. All 15

Table 2: Comparison of the optimized pulse duration (in $\mathrm{ms}$ ) with the duration of the PINS initial guess for all TSE examples.

\begin{tabular}{ccccccccc}
\multicolumn{4}{c}{ MB8 } & \multicolumn{2}{c}{ MB10 } & \multicolumn{2}{c}{ MB12 } & \multicolumn{2}{c}{ MB14 } \\
\hline $\begin{array}{c}\text { THK } \\
\mathrm{mm}\end{array}$ & $\begin{array}{c}\text { PINS } \\
\mathrm{ms}\end{array}$ & $\begin{array}{c}\text { opt } \\
\mathrm{ms}\end{array}$ & $\begin{array}{c}\text { PINS } \\
\mathrm{ms}\end{array}$ & $\begin{array}{c}\text { opt } \\
\mathrm{ms}\end{array}$ & $\begin{array}{c}\text { PINS } \\
\mathrm{ms}\end{array}$ & $\begin{array}{c}\text { opt } \\
\mathrm{ms}\end{array}$ & $\begin{array}{c}\text { PINS } \\
\mathrm{ms}\end{array}$ & $\begin{array}{c}\text { opt } \\
\mathrm{ms}\end{array}$ \\
\hline 2.0 & 10.640 & 2.742 & 9.460 & 2.856 & 8.810 & 2.728 & 8.710 & 2.809 \\
1.5 & 13.260 & 3.295 & 11.110 & 3.213 & 10.890 & 3.286 & 10.300 & 3.296 \\
1.0 & 18.040 & 3.958 & 15.170 & 4.122 & 14.460 & 4.120 & 14.110 & 3.996 \\
0.5 & 32.420 & 6.106 & 27.390 & 6.113 & 25.230 & 6.080 & 24.520 & 6.189 \\
\hline
\end{tabular}



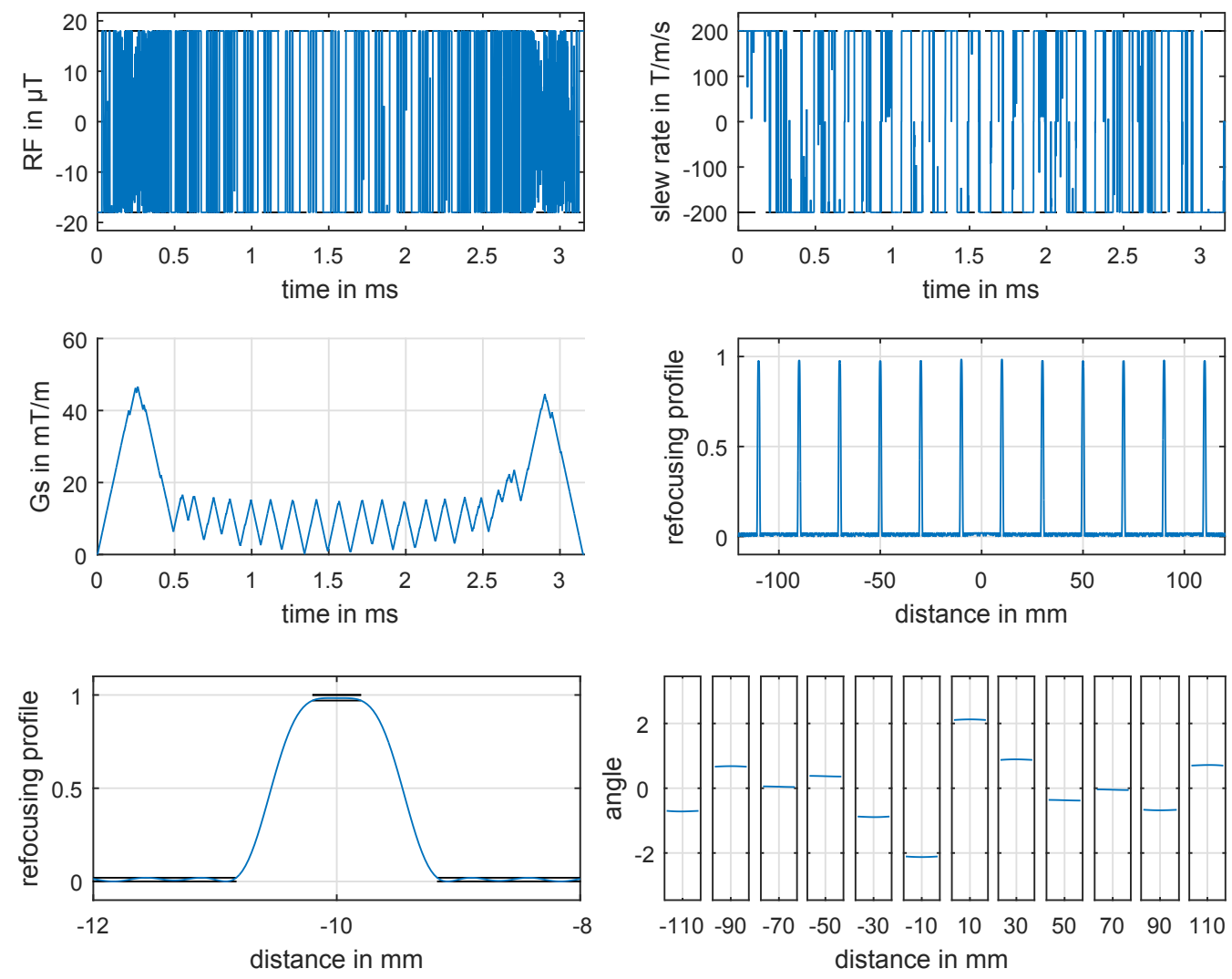

Figure 1: Optimization results and Bloch simulations for one representative TSE example. The first row shows the control variables $B_{1}$ amplitude and slew rate of Gs for the refocusing duration of $3.16 \mathrm{~ms}$. The second row depicts Gs shape, and the corresponding simulated refocusing profile $\left|b_{N_{t}}\right|^{2}$. The last row shows a detail zoom of one slice to see the refocusing profile together with the error corridor (black), and the phase $\operatorname{angle} \arg \left(b_{N_{t}}^{2}\right)$ per slice.

DIFF examples show very short pulse durations $2<T<3$ ms. More closely, the optimized durations increase slightly with decreasing THK and with increasing MB factor. The SAR constraints of all optimized DIFF examples are not active and do not restrict the temporal reduction. The case with the maximum SAR observed in the optimum was $\mathrm{MB}=4$ with $\mathrm{THK}=1 \mathrm{~mm}$ and a SAR of 3.16. In contrast, all TSE examples show an active SAR constraint in the optimum that limits a further temporal reduction. As a consequence, TSE examples with small slice thickness show a larger pulse duration of up to $6 \mathrm{~ms}$, and the optimized durations depend mainly on the slice thickness. For example, the RF pulses shown in Figure 2 have a SAR $\mathrm{e}_{\mathrm{e}}$ of $3.03 \mathrm{~W} \mathrm{~kg}^{-1}$ (DIFF optimized) and $3.19 \mathrm{~W} \mathrm{~kg}^{-1}$ (TSE optimized). The initial PINS pulses feature a lower SAR estimate $\left(1.47 \mathrm{~W} \mathrm{~kg}^{-1}\right.$ (DIFF init) and 2.46 $\mathrm{W} \mathrm{kg}^{-1}$ (TSE init)), mainly due to the much longer pulse duration. The 31 optimized pulses behind this Figure fulfill all constraints of the optimization model exactly, since the software rejects pulses with even minor violation in any constraint.

Figure 3 shows the optimized RF and Gs shapes for one DIFF and one TSE example using three different hardware constraints given in Supporting Table S2. Here, $\tau$ is set to the minimal gradient raster time of $10 \mu \mathrm{s}$ of the 3T MR scanner used later in the experimental validation. The computation times (scanner 2) of these pulses are approximately $2 \mathrm{~h}$ (DIFF) and 2.5h (TSE) using MPI on the hardware (16 CPU cores) described above.

Next, we investigated the influence of time-invariant $B_{0}$ and $B_{1}$ inhomogeneities on the two optimized pulses shown in Figure 1 and Supporting Figure S1. Bloch simulations were performed with an offresonance range of $\pm 200 \mathrm{~Hz}$ and a $B_{1}$ variation of $75-125 \%$. The results for the optimized pulses and 
DIFF

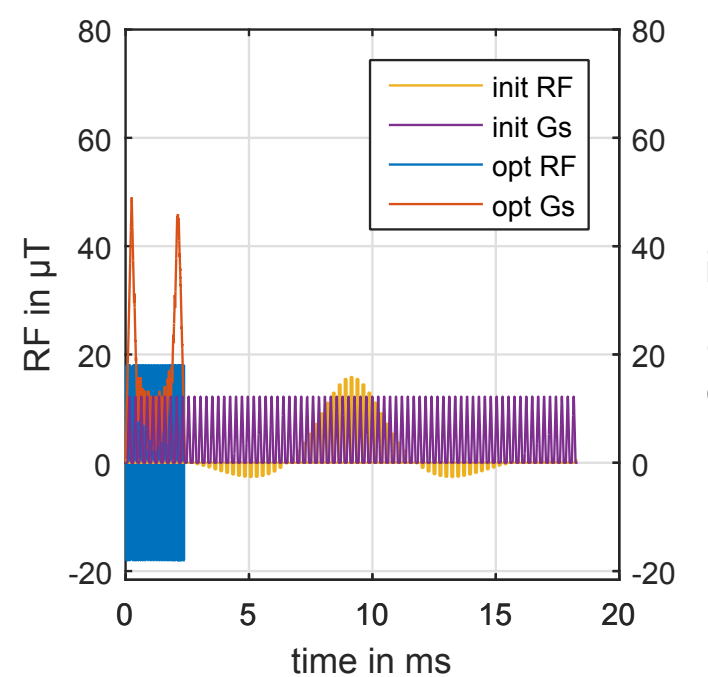

TSE

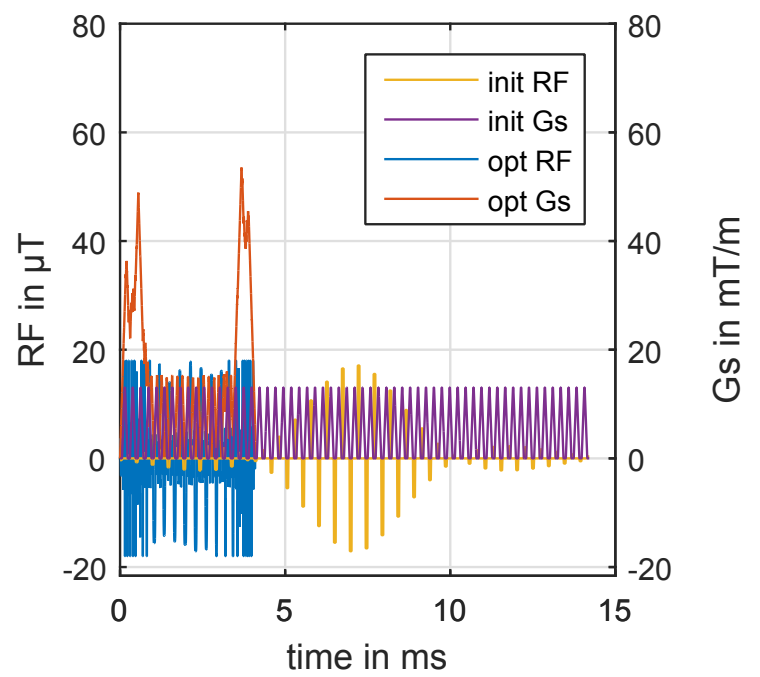

Figure 2: Comparison of the optimized results shown in Figure 1 and Supporting Figure S1 with the initial guess from PINS.
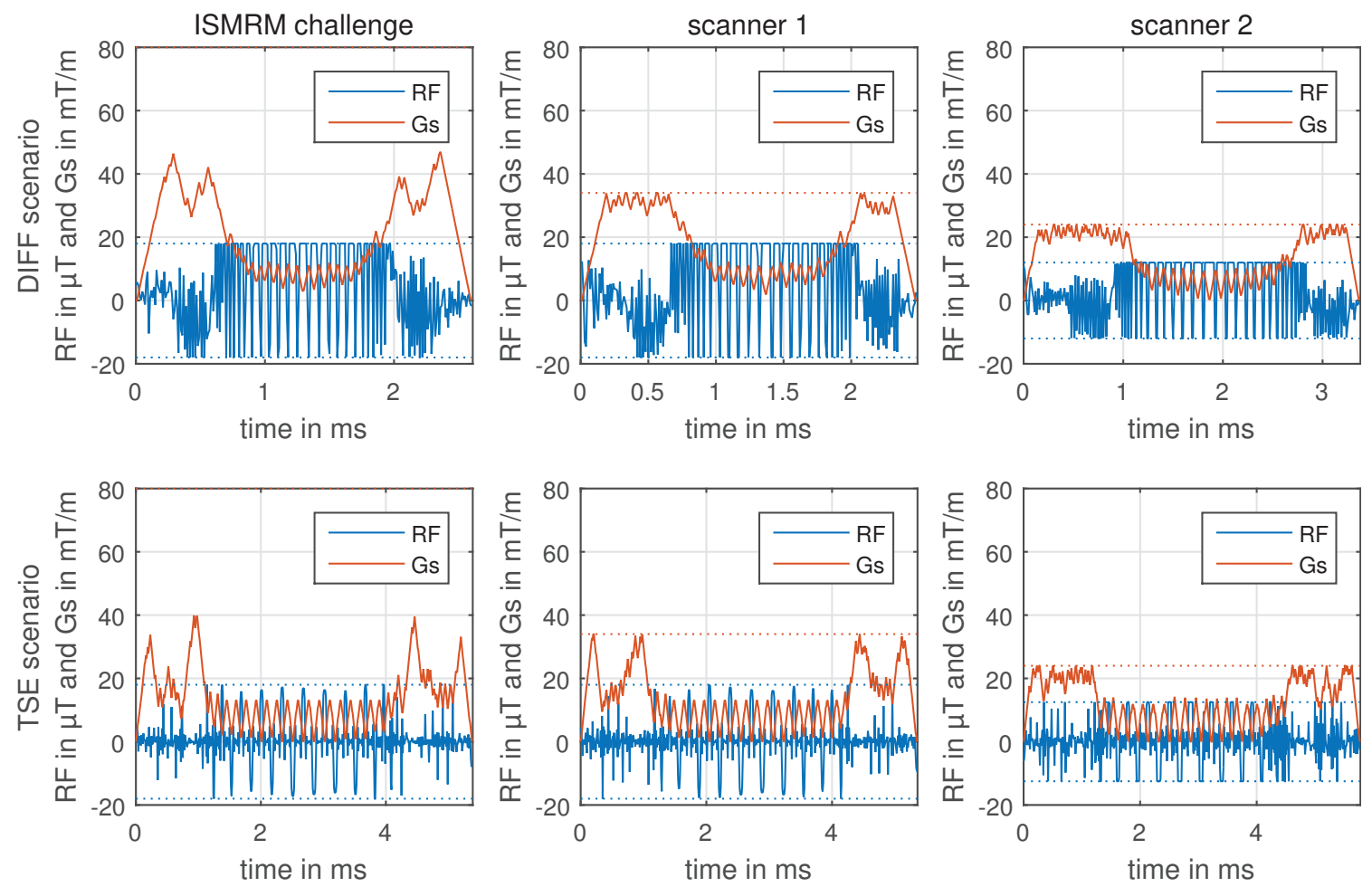

Figure 3: Optimized RF and Gs shapes for different hardware constraints (shown dotted) summarized in Supporting Table S2 (left: ISMRM challenge, middle: scanner 1, right: scanner 2) and a fixed temporal resolution of $10 \mu \mathrm{s}$. The upper row shows a DIFF pulse for 3 slices with a thickness of $1.75 \mathrm{~mm}$ and the lower row shows a TSE pulse for 12 slices with a thickness of $1 \mathrm{~mm}$. 

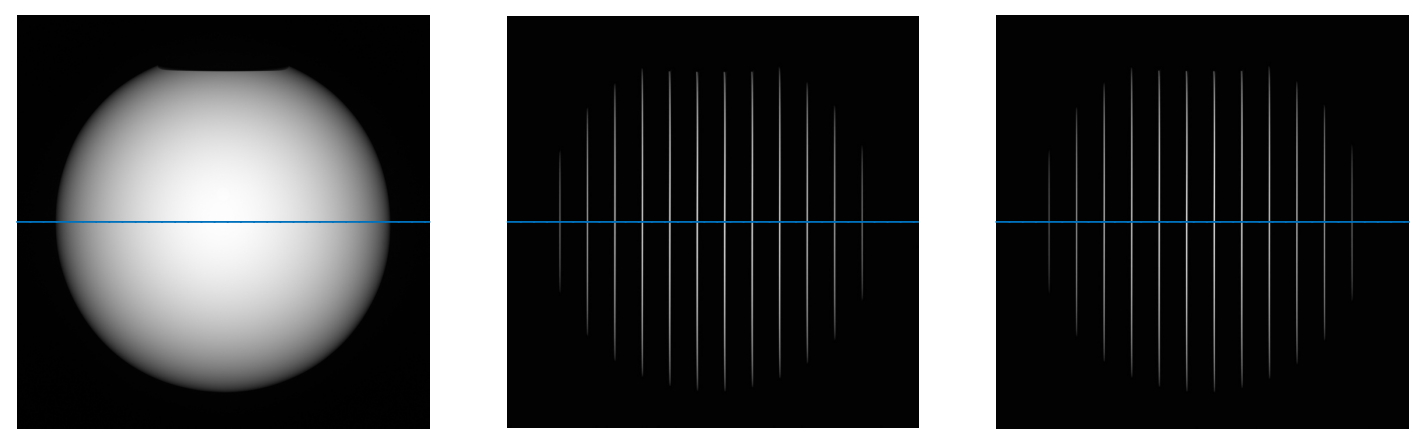

Figure 4: Reconstructed experimental spin echo data using a spherical oil phantom. Three different pairings are used: non-selective excitation and refocusing (left), slice-selective MB12 excitation and non-selective refocusing (middle), and slice-selective MB12 excitation and optimized refocusing (right). The intersection is shown in Figure 5 in detail.

the corresponding initial PINS pulse are depicted in Supporting Figure S2.

The optimized results do not change significantly w.r.t. the time resolution of the PINS initial guess or the initialization of the objective parameters, as long as the final time sampling rate is the same. In contrast, a coarser time discretization at the end generally leads to an increased pulse duration. For instance, the shortest diffusion candidate in Figure 3 optimized for a temporal discretization of $\tau=10$ $\mu$ s is about $17 \%$ longer $(T=2.61 \mathrm{~ms})$ than the optimized candidate with a temporal discretization of $0.625 \mu$ s shown in Table 1 with $T=2.155 \mathrm{~ms}$.

\subsection{Experiments}

Figure 4 shows the image reconstructions (magnitude) of the three SE experiments using a spherical oil phantom in the above mentioned 3T MR scanner. The optimized refocusing is based on the RF and Gs shapes of Figure 3 ("scanner 2"). The measured and reconstructed magnitude signal along the blue intersection line is plotted in detail in Figure 5 for the two slice-selective SE experiments.

There, the first and third row show the median of the high resolution DIFF and TSE phantom measurements, whereas the second, fourth and fifth row display zoomed images of the slices displaying both the median (solid) and the measurements points (crosses). The median is computed out of five individual measurements with minor noise variations. There is a good agreement between the different SE experiments with a small signal reduction for the outermost OC TSE slices, visible in the lowest right plot of Figure 5. The larger span in slice direction of the TSE example $(240 \mathrm{~mm})$ led to a comparable slice shift for both, the non-selective and optimized refocused data.

Figure 6 shows the intersection of the optimized SE experiment (median) normalized by the median of the non-selective SE experiment (shown for TSE in Figure 4). This normalization corrects for spatial $B_{1}$ transmit/receive and signal variations resulting from the spherical phantom. The normalized data points were compared to the numerical Bloch simulations of the used excitation and optimized refocusing (solid).

\section{Discussion}

In addition to strict hardware and safety constraints, the minimal excitation or refocusing duration is a critical and important parameter. Specifically in the context of SMS imaging, RF pulses tend to 

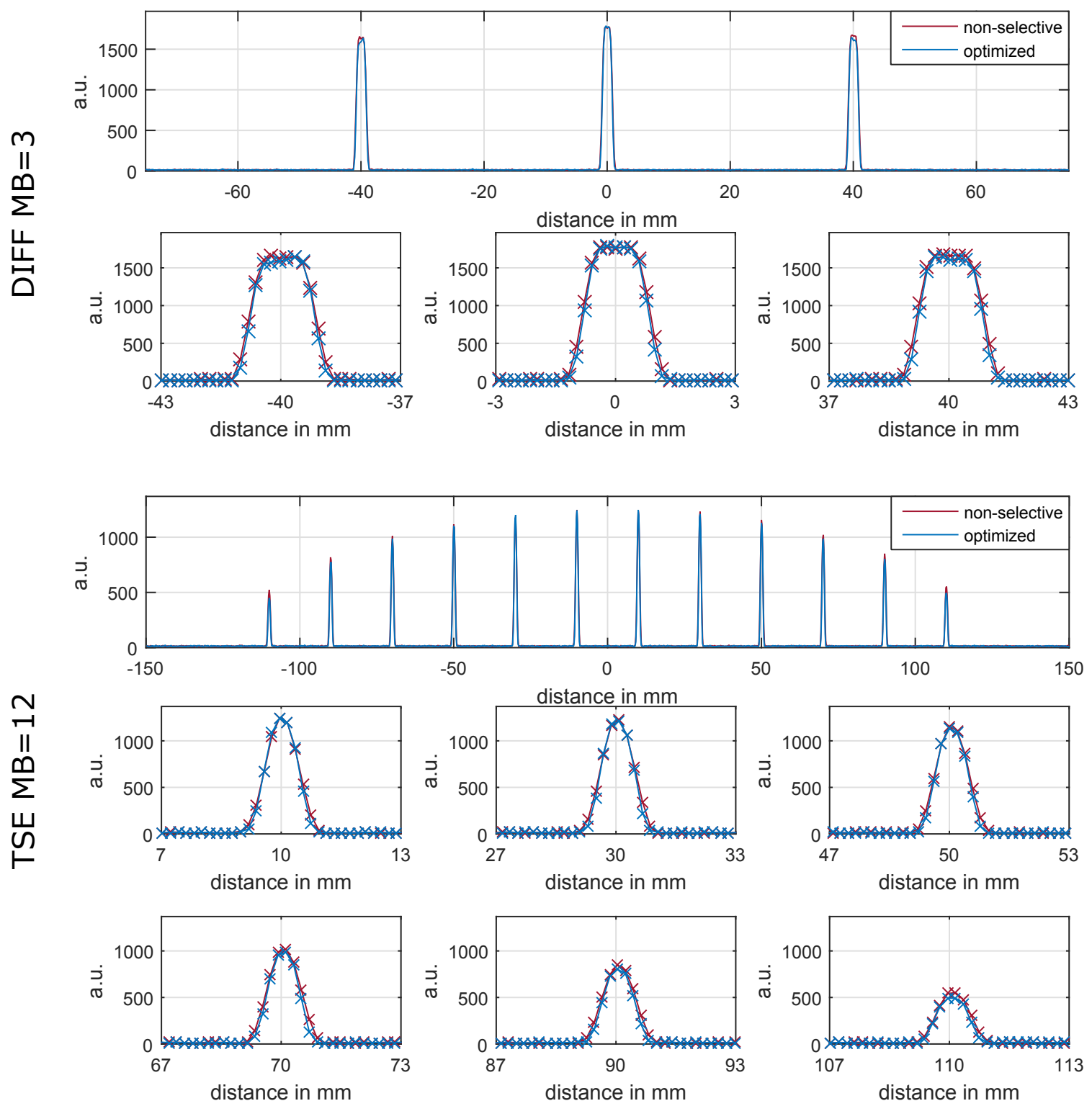

Figure 5: Reconstructed experimental spin echo data for DIFF MB $=3$ and TSE MB $=12$ refocusing with a slice thickness of $1.75 \mathrm{~mm}$ and $1 \mathrm{~mm}$ using a spherical phantom. Two different pairings are used: slice-selective excitation and non-selective refocusing (non-selective), and sliceselective excitation and optimized refocusing (optimized). Row one and three show the median of four individual measurements per curve. Row two, four and five show a zoom into the slices where the measurement points are additionally plotted (crosses). 

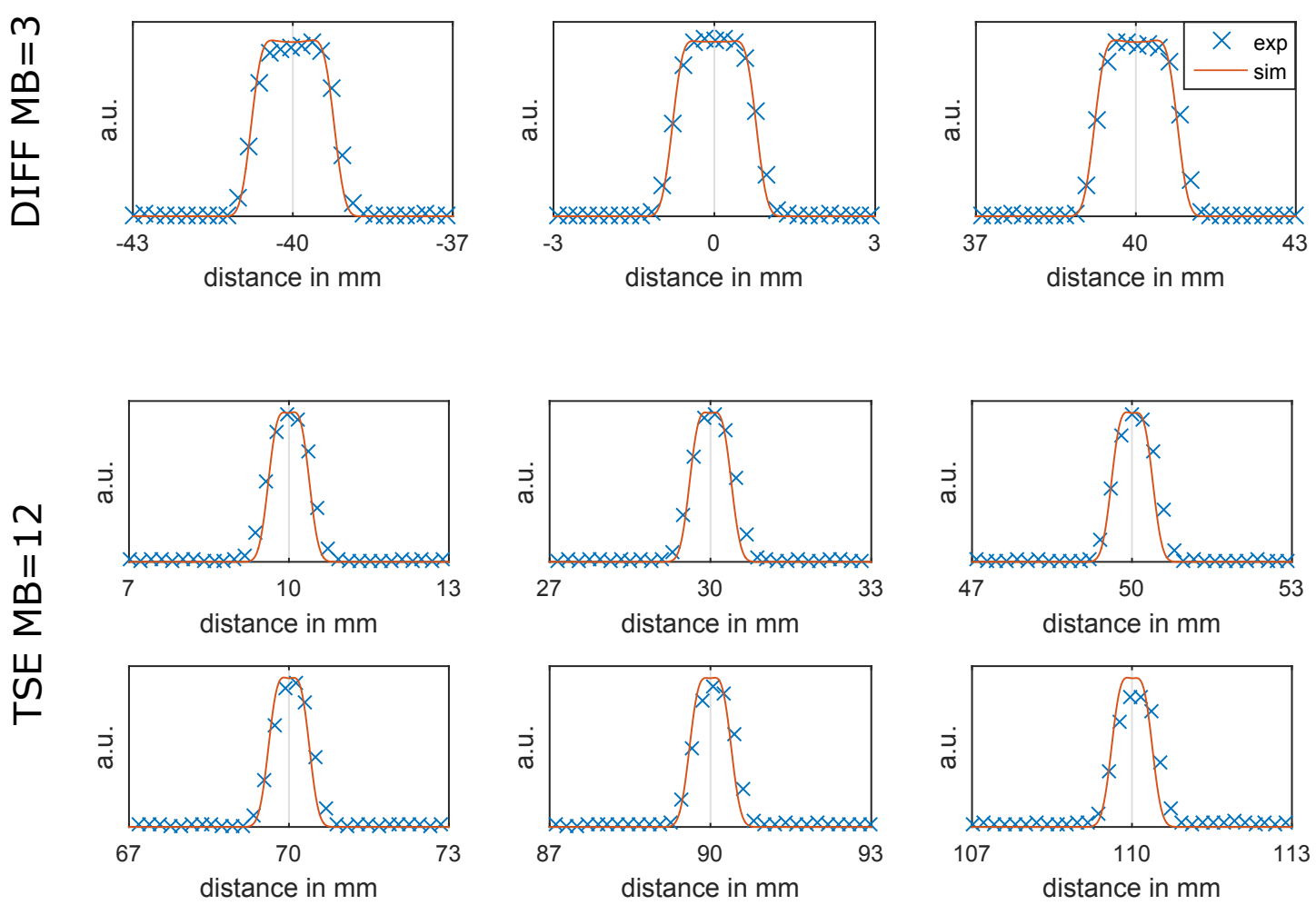

Figure 6: Comparison of the experimental data (exp) with optimized refocusing normalized by a fully non-selective SE measurement and the Bloch simulations (sim) for slice-selective excitation and optimized DIFF $\mathrm{MB}=3$ and TSE $\mathrm{MB}=12$ refocusing.

have unacceptable long pulse durations limiting the applications and capabilities of SMS imaging. This work presented a time optimal control method, that, together with constrained optimization [36], can be applied to drastically reduce the pulse duration of SMS refocusing pulses while still fulfilling the hardware constraints and slice profile accuracy. The methods were tested on the 31 examples of the ISMRM Challenge on RF pulse design [18]. Compared to the given initialization of the ISMRM challenge (conventional superposed frequency shifted candidates for the diffusion cases, PINS for the TSE cases) the sum of the pulse durations for the 31 examples was reduced from $520.4 \mathrm{~ms}$ down to $102.4 \mathrm{~ms}$, which is a reduction of $80.3 \%$.

The proposed time optimal control method was tested for different problem parameters (Supporting Table S1) and constraints (Supporting Table S2). It robustly delivered pulses that exploit the allowed error bands to significantly shorten the pulse duration. This robustness w.r.t. the parameters and initializations is a consequence of using a trust-region globalization of the Newton-type method in the lower level problem. During the Challenge we tested the optimizer on different initializations computed by frequency shifted superposition, phase modulation and PINS. In all cases the pulses were shortened significantly. The shortest pulse durations were in most cases obtained for PINS based initial pulses, however, other educated guesses may outperform the presented results. We note that different initializations can be tested by the user in the published software.

The simulations of the optimized SMS pulses show accurate refocusing profiles with errors below the prescribed error bounds. Both, the treatment of the control constraints (i.e. peak $B_{1}$ amplitude and the peak slew rate of Gs) by a semismooth quasi-Newton method and the treatment of the state constraints (profile accuracy, phase constraints, amplitude of Gs) by an $L^{p}$ penalization with an iteratively increased $p \rightarrow \infty$ allowed for full exploitation of the inequality constraints [36]. The precise derivative information (exact discrete derivatives using adjoint calculus, second-order method) enabled additional progress in reducing the pulse duration, when many of the constraints were already active. In contrast to the SAR minimization for fixed pulse duration in [36] the RF amplitude constraint 
is much more important in the time optimal case. In particular, the optimized controls in Figure 1 and Supporting Figure $S_{1}$ show that (if the SAR and peak gradient constraint are not active) all the constraints are active wherever it is possible, resulting in equiripple error distributions and bang-bang controls. This is a typical behavior of solutions of pure time optimal control problems, and it underlines the local optimality of the presented results. With stronger constraints on the SAR or the peak gradient amplitude, optimized durations increase. In this case the optimal RF amplitude is at its bounds only in certain points during the time interval, see Figure 3.

In all examples, the pulse duration can be further decreased by a temporal refinement at the cost of an increased computational effort. For example the diffusion case shown in Figure 3 yielded a minimum pulse duration of $2.61 \mathrm{~ms}$ for a typical time grid $(\tau=10 \mu \mathrm{s})$ and of $2.155 \mathrm{~ms}$ after four further refinement steps $(\tau=2.5 \mu \mathrm{s})$. For the SE measurements the temporal refinement was done only up to the minimal gradient raster time of the particular MR scanner.

All optimized examples are designed and evaluated for a distinct FOV. We would like to mention here that both the PINS initial and optimized pulses create refocusing slices outside the FOV of interest. If this is unacceptable, the use of different initial guesses such as superposition pulses allows to increase the FOV and further restricts the refocusing profiles.

Optimized pulse durations of under $3 \mathrm{~ms}$ were observed throughout all diffusion examples, see Table 1 . Here, the SAR was never at its bounds, mainly because of the low pulse rate. The maximum amplitude $g_{\max }$ for the Gs amplitude was only reached in 3 of the 15 examples. In contrast, all optimized RF amplitudes and gradient slew rates were at their bounds almost everywhere with small exceptions around the two time points of maximum gradient amplitude.

For the TSE examples, the initial PINS pulses already combine a good refocusing profile with a small $\mathrm{RF}$ power that is exploited in the temporal reduction. Due to a higher pulse rate, the SAR constraint has a strong influence on the pulse duration here and is the main limiting factor for the temporal reduction. All 16 cases show an active SAR constraint in the optimum and outperform the initial PINS pulses in terms of the required refocusing duration. For the case given in Figure 1, the optimizer computes an admissible pulse candidate with a minimal pulse duration of $3.155 \mathrm{~ms}$ without a SAR constraint, compared to 4.120 ms with a SAR constraint, see Figure 2 and Table 2, of $3.2 \mathrm{~W} \mathrm{~kg}^{-1}$ - a factor of roughly $25 \%$. This results in less exploited RF shape where the RF envelope differs from the block shape of examples without an active power constraint, e.g. the DIFF MB5 case shown in Figure 2. As a consequence of the active SAR constraint, the minimum durations turn out to be independent of the MB factor, see Table 2. Instead, they mainly depend on the slice thickness.

In total we applied the proposed optimization method to design SMS refocusing pulses with a wide range of MB factors (3-14) and THK (0.5-2 mm). All optimized pulses show a dramatically reduced pulse duration, on average by $87.1 \%$ for the diffusion and by $74.5 \%$ for the TSE examples, which allows significant reduction of the minimal TE of both, diffusion and TSE/RARE sequences.

Compared to state-of-the-art minimum duration design methods such as root-flip design [41], the achieved reduction of the proposed method is still significantly higher. For instance, the DIFF pulse used in the experiment (see Figure 3 ) is $57.4 \%$ shorter than a root-flipped pulse ( $T=6.11 \mathrm{~ms}$ ) designed with equivalent design parameters (512 time-points with $\tau=11.93 \mu \mathrm{s}, M B=3, T B P=4, T H K=1.75$ $\mathrm{mm}, r_{\max }=12.5 \mu \mathrm{T}$ and a refocusing error of 0.02 out-of-slice and 0.03 in-slice).

Comparison of DIFF examples without a distinct phase constraint with the TSE examples designed with a pointwise phase constraint of the refocusing slices revealed that the additional constraint only has a minor effect on the overall pulse duration. Although this is not shown in this work, the phase constraint can obviously be changed to treat all slices at once to comply with the CPMG condition [10, 31].

With the current CPU based MPI implementation the pulses need to be precomputed and provided on the MR scanner. To reduce the current computation time, a speed-up of a factor 31 has been reported by using GPU parallelization [9]. Both optimized SE measurements were in a good accordance with the non-selective refocusing. 
For comparison of simulated and measured slice profiles, the transmit/receive sensitivity variations and spatial signal differences arising from the used spherical oil phantom were removed by normalization of the measurements with a fully non-selective SE reference scan. Then, comparisons between the normalized slice profiles with optimized refocusing and the Bloch simulations were performed (Figure 6).

In general, rapidly-varying RF pulses can be distorted by the limited bandwidth of the RF system [17]. To reduce this RF distortion, we design real valued RF pulses that are less prone to RF distortions [2]. Additionally we used a time grid of $10 \mu \mathrm{s}, 400$ times the minimal RF duration of $25 \mathrm{~ns}$. Therefore we do not expect significant alterations of the RF chain on the optimized RF shapes. This is supported by the measured slice profiles. The question, whether time-optimal complex-valued RF pulses can outperform the real-valued ones will be part of future work. For systems with different RF specifications or for the optimization of complex RF pulses, an additional constraint on the RF slew rate, analogous to the presented Gs slew rate constraint, could be included in the optimization.

The question arises how the optimized pulses perform in the presence of gradient imperfections. Besides an identical slice shift for both refocusing examples (see Figure 5) the slices of the optimized refocusing are attenuated with the distance to the isocenter. After a manual shim there are only minor $B_{0}$ inhomogeneities in the phantom and the slice shift mainly results from non-linear gradients at the boundary of the field of view. The minor signal attenuations of the outermost slices are likely caused by gradient distortions $[1,43]$. The inclusion of gradient and amplifier imperfections for different MR systems in the optimization will be future work.

An additional gradient echo phase scan (not shown) showed a slightly asymmetric $B_{0}$ field that explains spatial signal differences between data from non-selective excitation and data from slice-selective excitation. However, these residual $B_{0}$ inhomogeneities are not strong enough to explain the observed signal loss. The robustness of the optimized pulses w.r.t. $B_{0}$ and $B_{1}$ inhomogeneities was investigated in simulations in comparison to the initial pulses (Supporting Figure $S_{2}$ ). In general the slice displacement for both optimized cases are lower compared with the initial PINS pulses. This is mainly due to the heavily decreased pulse duration which reduces the $B_{0}$ sensitivity. Due to a variable k-space velocity of the optimized examples, the refocusing profiles are thinned out with increasing off-resonance, but remain stable below $\pm 100 \mathrm{~Hz}$. In the context of $B_{1}$ inhomogeneities, all examples share the principle that the thickness of the refocusing profile is broadened for lower $B_{1}$ scaling and thinned out for a higher $B_{1}$ scaling similar to other studies [25]. Depending on the application, an inclusion of $B_{0} / B_{1}$ robustness into the optimization framework will be focus of future work.

\section{Conclusions}

The proposed time optimal design method yields optimized SMS refocusing pulses for clinical sequences with very short pulse durations with respect to representative physical constraints given by current scanner hardware. The time optimal refocusing pulses will be beneficial for a broad range of SMS applications such as diffusion and spin echo based sequences to reduce the echo spacing and increase the signal quality in terms of amplitude and robustness to motion.

\section{Acknowledgments}

Partial support from "SFB F32-N18" and BioTechMed-Graz are gratefully acknowledged. This work was in part supported by the ERC advanced grant 668998 (OCLOC) under the EU's H2Ozo research program. The authors thank Christian Clason from the University of Duisburg-Essen for many fruitful discussions on the topic of the paper. 


\section{References}

[1] S. Abo Seada, J. V. Hajnal, and S. J. Malik. A simple optimisation approach to making time efficient VERSE-multiband pulses feasible on non-ideal gradients. In Proceedings of the 25th Annual Meeting of ISMRM, page 5059, 2017.

[2] S. Abo Seada, A. N. Price, J. V. Hajnal, and S. J. Malik. Optimized amplitude modulated multiband RF pulse design. Magn Reson Med, 78(6):2185-2193, 2017. URL: http://dx.doi.org/10.1002/ mrm. 26610, doi : 10.1002/mrm. 26610.

[3] C. S. Aigner, C. Clason, A. Rund, and R. Stollberger. Efficient high-resolution RF pulse design applied to simultaneous multi-slice excitation. $\mathcal{F}$ Magn Reson, 263:33-44, 2016. doi: http://dx. doi.org/10.1016/j.jmr.2015.11.013.

[4] C. K. Anand, A. D. Bain, A. T. Curtis, and Z. Nie. Designing optimal universal pulses using second-order, large-scale, non-linear optimization. F Magn Reson, 219:61-74, 2012. doi: doi: 10.1016/j . jmr. 2012.04.004.

[5] E. J. Auerbach, J. Xu, E. Yacoub, S. Moeller, and K. Uğurbil. Multiband accelerated spin-echo echo planar imaging with reduced peak RF power using time-shifted RF pulses. Magn Reson Med, 69(5):1261-1267, 2013. doi: 10.1002/mrm. 24719.

[6] M. Barth, F. Breuer, P. Koopmans, D. Norris, and B. Poser. Simultaneous multislice (SMS) imaging techniques. Magn Reson Med, 81:63-81, 2016. doi:10.1002/mrm. 25897.

[7] M. A. Bernstein, K. F. King, and X. J. Zhou. Handbook of MRI Pulse Sequences. Elsevier Academic Press, 2004. doi: http://dx. doi.org/10.1016/B978-012092861-3/50003-0.

[8] B. Bilgic, B. Gagoski, S. Cauley, A. Fan, J. Polimeni, P. Grant, L. Wald, and K. Setsompop. WaveCAIPI for highly accelerated 3D imaging. J Magn Reson, 73(6):2152-2162, 2015. doi : 10.1002/ mrm. 25347.

[9] M. Bödenler, C. S. Aigner, A. Rund, C. Clason, and R. Stollberger. Fast optimization of RF excitation. In Proceedings of the 29th Annual Meeting of ESMRMB, pages 247-400, 2016.

[10] H. Y. Carr and E. M. Purcell. Effects of diffusion on free precession in nuclear magnetic resonance experiments. Phys Rev, 94:630-638, May 1954. doi:10.1103/PhysRev.94.630.

[11] L. Chen, A. T. Vu, J. Xu, S. Moeller, K. Uğurbil, E. Yacoub, and D. Feinberg. Evaluation of highly accelerated simultaneous multi-slice EPI for fMRI. NeuroImage, 104:452-459, 2015. doi: http: //dx.doi.org/10.1016/j. neuroimage.2014.10.027.

[12] G. Ciaramella and A. Borzì. SKRYN: A fast semismooth-Krylov-Newton method for controlling Ising spin systems. Comput Phys Commun, 190:213-223, 2015. doi:http://dx.doi.org/10. 1016/j.cpc.2015.01.006.

[13] S. Conolly, D. Nishimura, and A. Macovski. Optimal control solutions to the magnetic resonance selective excitation problem. IEEE Trans Med Imaging, MI-5(2):106-115, 1986.

[14] S. Conolly, D. Nishimura, A. Macovski, and G. Glover. Variable-rate selective excitation. 7 Magn Reson, 78(3):440-458, 1988. doi : http://dx. doi .org/10.1016/0022-2364(88) 90131-X.

[15] C. Eichner, L. L. Wald, and K. Setsompop. A low power radiofrequency pulse for simultaneous multislice excitation and refocusing. Magn Reson Med, 72(4):949-958, 2014. doi : 10 . 1002/mrm. 25389.

[16] B. A. Gagoski, B. Bilgic, C. Eichner, H. Bhat, P. E. Grant, L. L. Wald, and K. Setsompop. RARE/turbo spin echo imaging with simultaneous multislice Wave-CAIPI. Magn Reson Med, 73(3):929-938, 2015. doi: 10.1002/mrm. 25615. 
[17] W. A. Grissom, A. B. Kerr, P. Stang, G. C. Scott, and J. M. Pauly. Minimum envelope roughness pulse design for reduced amplifier distortion in parallel excitation. Magn Reson Med, 64(5):1432-1439, 2010. URL: http://dx.doi.org/10.1002/mrm.22512, doi : 10.1002/mrm. 22512.

[18] W. A. Grissom, K. Setsompop, S. A. Hurley, J. Tsao, J. V. Velikina, and A. A. Samsonov. Advancing RF pulse design using an open-competition format: Report from the 2015 ISMRM challenge. Magn Reson Med, pages 1-10, 2016. doi : 10.1002/mrm. 26512.

[19] W. A. Grissom, D. Xu, A. B. Kerr, J. A. Fessler, and D. C. Noll. Fast large-tip-angle multidimensional and parallel RF pulse design in MRI. IEEE Trans Med Imaging, 28(10):1548-1559, 2009. doi: 10.1109/TMI . 2009. 2020064.

[20] J. P. Haldar, Q. Fan, and K. Setsompop. Whole-brain quantitative diffusion MRI at $660 \mu \mathrm{m}$ resolution in 25 minutes using gSlider-SMS and SNR-enhancing joint reconstruction. In Proceedings of the 24th Annual Meeting of ISMRM, page 0102, 2016.

[21] B. A. Hargreaves, C. H. Cunningham, D. G. Nishimura, and S. M. Conolly. Variable-rate selective excitation for rapid MRI sequences. Magn Reson Med, 52(3):590-597, 2004. doi : 10. 1002/mrm. 20168.

[22] H. Hermes and J. P. Lasalle. Functional Analysis and Time Optimal Control. Mathematics in Science and Engineering. Academic Press, New York, 1969. URL: http://books.google.at/ books?id=U20pf6C83B8C.

[23] M. Hintermüller and K. Kunisch. Stationary Optimal Control Problems with Pointwise State Constraints, volume 72 of Lecture Notes in Computational Science and Engineering. Springer, Berlin, 2009.

[24] A. M. Huettner, N. J. Mickevicius, A. Ersoz, K. M. Koch, L. T. Muftuler, and A. S. Nencka. Wavelet domain radiofrequency pulse design applied to magnetic resonance imaging. PLOS ONE, 10(10):117, 10 2015. doi: 10.1371/journal . pone. 0141151.

[25] M. A. Janich, R. F. Schulte, M. Schwaiger, and S. J. Glaser. Robust slice-selective broadband refocusing pulses. F Magn Reson, 213(1):126-135, 2011. URL: http://www. sciencedirect. com/science/ article/pii/S1090780711003375, doi:http://doi.org/10.1016/j.jmr.2011.09.025.

[26] E. Jaynes. Matrix treatment of nuclear induction. Physical Review, 98(4):1099-1105, 1955.

[27] N. Khaneja, T. Reiss, C. Kehlet, T. Schulte-Herbrüggen, and S. J. Glaser. Optimal control of coupled spin dynamics: design of NMR pulse sequences by gradient ascent algorithms. I Magn Reson, 172(2):296-305, 2005. doi: 10.1016/j . jmr. 2004 .11.004.

[28] N. Kobayashi, K. Ugurbil, and X. Wu. Shortening nonlinear phase multiband refocusing pulses with VERSE. In Proceedings of the 24th Annual Meeting of ISMRM, page 3253, 2016.

[29] K. Kunisch, K. Pieper, and A. Rund. Time optimal control for a reaction diffusion system arising in cardiac electrophysiology - a monolithic approach. ESAIM Math Model Num, 50(2):381-414, 2016. URL: dx.doi.org/10.1051/m2an/2015048, doi:10.1051/m2an/2015048.

[3o] D. J. Larkman, J. V. Hajnal, A. H. Herlihy, G. A. Coutts, I. R. Young, and G. Ehnholm. Use of multicoil arrays for separation of signal from multiple slices simultaneously excited. $\mathcal{F}$ Magn Reson Imaging, 13(2):313-317, 2001. doi : 10 . 1002/1522 - 2586 (200102) 13: 2<313: : AID - JMRI1045>3 . 0 . C0 ; 2 -W.

[31] S. Meiboom and D. Gill. Modified spin-echo method for measuring nuclear relaxation times. Rev Sci Instrum, 29(8):688-691, 1958. URL: http://dx. doi .org/10.1063/1.1716296, doi : 10.1063/ 1.1716296.

[32] S. Mueller. Multifrequency selective RF pulses for multislice MR imaging. Magn Reson Med, 6(3):364-371, 1988. doi:10.1002/mrm . 1910060315. 
[33] D. G. Norris, P. J. Koopmans, R. Boyacioğlu, and M. Barth. Power independent of number of slices (PINS) radiofrequency pulses for low-power simultaneous multislice excitation. Magn Reson Med, 66(5):1234-1240, 2011. doi : 10.1002/mrm. 23152.

[34] J. Pauly, P. Le Roux, D. Nishimura, and A. Macovski. Parameter relations for the Shinnar-Le Roux selective excitation pulse design algorithm. IEEE Trans Med Imaging, 10(1):53-65, 1991. doi: 10.1109/42.75611.

[35] T. Reese, O. Heid, R. Weisskoff, and V. Wedeen. Reduction of eddy-current-induced distortion in diffusion MRI using a twice-refocused spin echo. Magn Reson Med, 49(1):177-182, 2003. URL: http://dx.doi.org/10.1002/mrm. 10308, doi:10.1002/mrm. 10308.

[36] A. Rund, C. Aigner, K. Kunisch, and R. Stollberger. Magnetic resonance RF pulse design by optimal control with physical constraints. IEEE Trans Med Imaging, page early access: https://doi.org/10.1109/TMI.2017.2758391, 2017. URL: https://doi.org/10.1109/TMI.2017. 2758391, doi:10.1109/TMI . 2017.2758391.

[37] A. Rund, C. Aigner, K. Kunisch, and R. Stollberger. RF pulse design by optimal control with physical constraints. In Proceedings of the 25th Annual Meeting of ISMRM, page 1435, 2017.

[38] K. Setsompop, B. Bilgic, A. Nummenmaa, Q. Fan, S. F. Cauley, S. Huang, I. Chatnuntawech, Y. Rathi, T. Witzel, and L. L. Wald. Slice dithered enhanced resolution simultaneous multislice (SLIDER-SMS) for high resolution (70o um) diffusion imaging of the human brain. In Proceedings of the 23th Annual Meeting of ISMRM, page 0339, 2015.

[39] K. Setsompop, J. Cohen-Adad, B. A. Gagoski, T. Raij, A. Yendiki, B. Keil, V. J. Wedeen, and L. L. Wald. Improving diffusion MRI using simultaneous multi-slice echo planar imaging. NeuroImage, 63(1):569-580, 2012. doi : 10.1016/j . neuroimage. 2012 .06.033.

[40] K. Setsompop, B. A. Gagoski, J. R. Polimeni, T. Witzel, V. J. Wedeen, and L. L. Wald. Blippedcontrolled aliasing in parallel imaging for simultaneous multislice echo planar imaging with reduced g-factor penalty. Magn Reson Med, 67(5):1210-1224, 2012. doi : 10. 1002/mrm. 23097.

[41] A. Sharma, M. Lustig, and W. A. Grissom. Root-flipped multiband refocusing pulses. Magn Reson Med, 75(1):227-237, 2016. doi : 10.1002/mrm. 25629.

[42] T. E. Skinner, N. I. Gershenzon, M. Nimbalkar, W. Bermel, B. Luy, and S. J. Glaser. New strategies for designing robust universal rotation pulses: Application to broadband refocusing at low power. J Magn Reson, 216:78-87, 2012. doi:10.1016/j . jmr .2012 .01.005.

[43] S. J. Vannesjo, M. Haeberlin, L. Kasper, M. Pavan, B. J. Wilm, C. Barmet, and K. P. Pruessmann. Gradient system characterization by impulse response measurements with a dynamic field camera. Magn Reson Med, 69(2):583-593, 2013. URL: http://dx. doi . org/10.1002/mrm. 24263, doi: $10.1002 / \mathrm{mrm} .24263$.

[44] M. Vinding, I. Maximov, Z. Tošner, and N. Nielsen. Fast numerical design of spatial-selective RF pulses in MRI using Krotov and quasi-Newton based optimal control methods. F Chem Phys, 137(5), 2012. doi:10.1063/1.4739755.

[45] E. Wong. Optimized phase schedules for minimizing peak RF power in simultaneous multi-slice RF excitation pulses. In Proceedings of the 2oth Annual Meeting of ISMRM, page 2209, 2012. 


\section{Supporting Information}
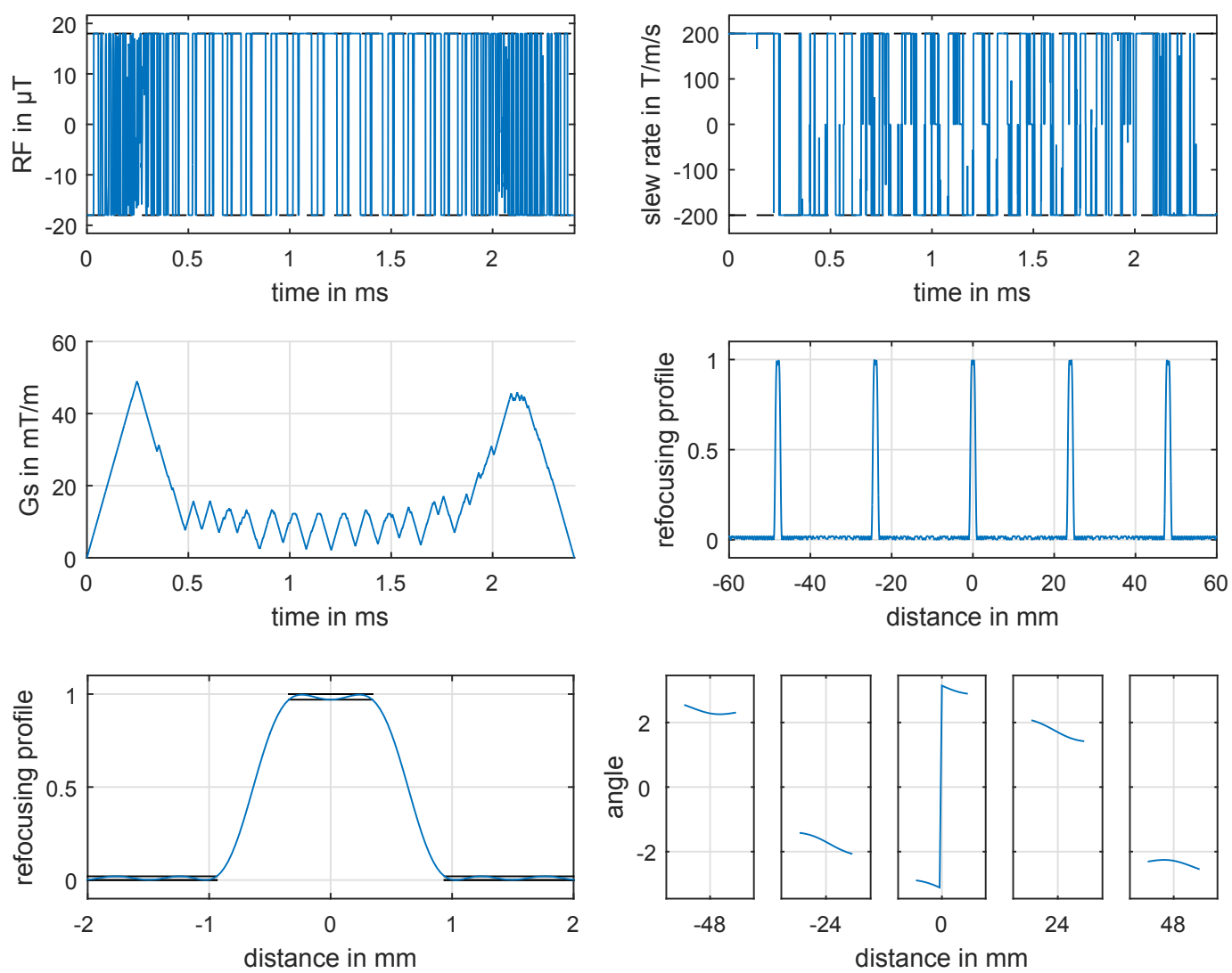

Supporting Figure S1: Optimization results and Bloch simulations for a representative diffusion example. The first row shows the control variables $B_{1}$ amplitude and slew rate of Gs with a total duration of $2.404 \mathrm{~ms}$. The second row depicts Gs shape, and the corresponding simulated refocusing profile $\left|b_{N_{t}}\right|^{2}$. The last row shows a detail zoom of one slice to see the refocusing profile together with the error corridor (black), and the phase angle $\arg \left(b_{N_{t}}^{2}\right)$ per slice.

Supporting Table S1: Overview of the problem parameters for TSE and diffusion (DIFF).

\begin{tabular}{lcccccc}
\hline $\begin{array}{c}\text { MB factor } \\
\text { [a.u.] }\end{array}$ & $\begin{array}{c}\text { FOV } \\
\mathrm{mm}\end{array}$ & $\begin{array}{c}\text { THK } \\
\mathrm{mm}\end{array}$ & $\begin{array}{c}\text { TBP } \\
\text { [a.u.] }\end{array}$ & $\begin{array}{c}f_{p} \\
1 / \mathrm{s}\end{array}$ & $\begin{array}{c}\text { phase } \\
\text { [a.u.] }\end{array}$ \\
\hline TSE & $8: 2: 14$ & 240 & $0.5: 0.5: 2.0$ & 3 & 54.55 & constant \\
DIFF & $3: 1: 5$ & 120 & $1.0: 0.25: 2.0$ & 4 & 16.67 & free \\
\hline
\end{tabular}

Supporting Table S2: Overview of the physical constraints used in the optimization.

\begin{tabular}{lccc}
\hline & $\begin{array}{c}G_{\max } \\
\mathrm{mT} / \mathrm{m}\end{array}$ & $\begin{array}{c}s_{\max } \\
\mathrm{mT} / \mathrm{m} / \mathrm{ms}\end{array}$ & $\begin{array}{c}r_{\max } \\
\mu \mathrm{T}\end{array}$ \\
\hline ISMRM challenge & 80.0 & 200 & 18.0 \\
scanner 1 & 34.0 & 200 & 18.0 \\
scanner 2 & 24.0 & 180 & 12.5 \\
\hline
\end{tabular}



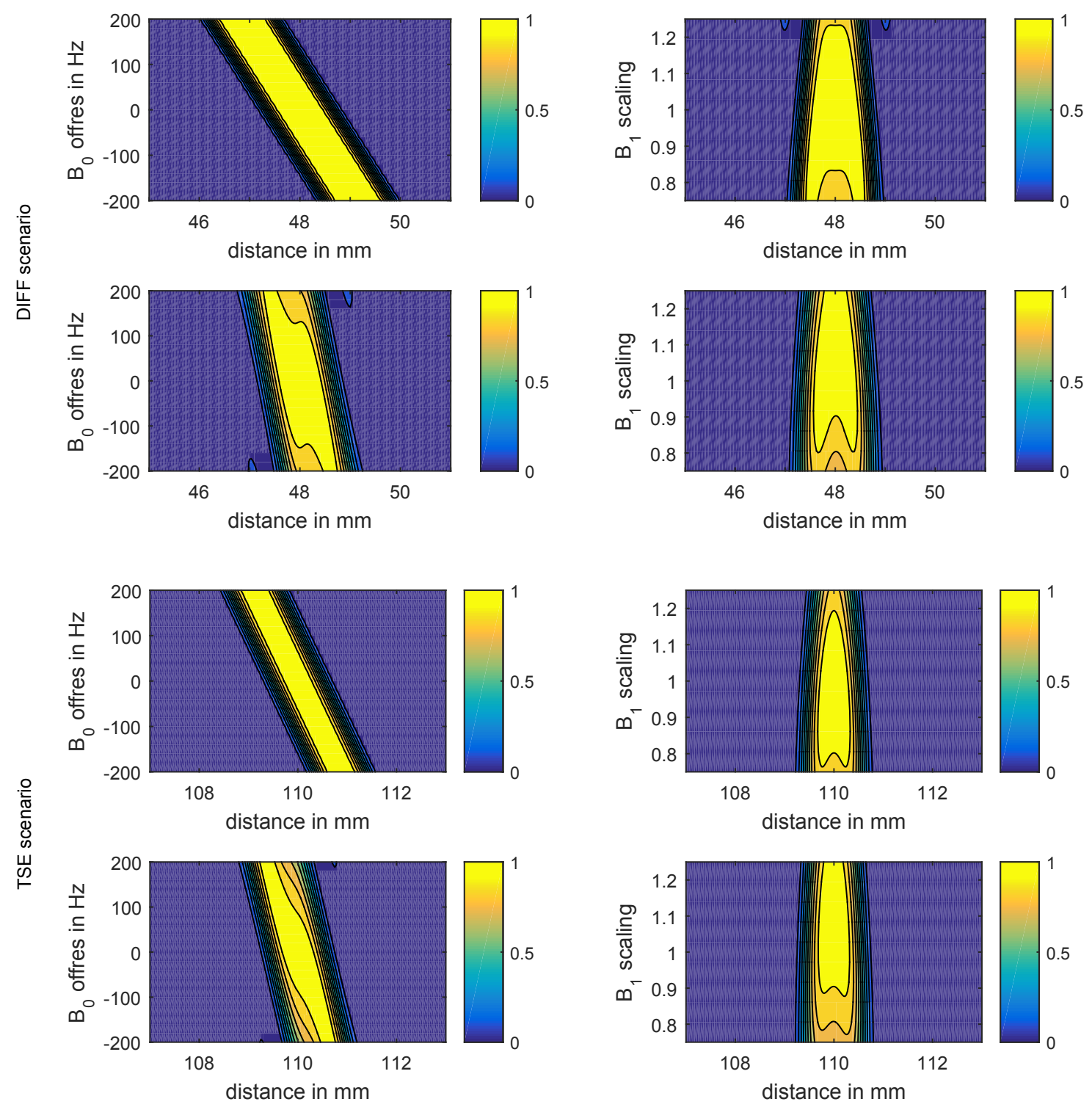

Supporting Figure S2: Simulated refocusing profiles $\left|b_{N_{t}}\right|^{2}$ (zoom to the outermost slice with comparable refocusing profiles across all individual slices) for a variation in the off-resonance and $B_{1}$ inhomogeneity for four different pulses: the PINS-based initial pulses (first and third row) and the optimized pulses shown in Supporting Figure $S_{1}$ (second row) and Figure 1 (fourth row), respectively. 